\title{
Use of the Asthma Control Questionnaire by the Asthma Clinical Research Network
}

\section{To the Editors:}

In our original report previously published in the European Respiratory Journal (ERJ) [1], the Asthma Clinical Research Network (ACRN) utilised an unauthorised modified version of the Asthma Control Questionnaire (ACQ) ${ }^{\#}$ administered over the telephone to demonstrate that the severity of a cold measured within the first 2 days was a predictor of subsequent worsening of asthma control. We subsequently stated this fact in an Erratum published in ERJ [2], and planned to conduct studies that would determine the impact of our modifications on the interpretation of our study.

The modified ACQ utilised in our study was a six-item modified version of a validated shortened version of the ACQ [3]; a question was also added to the beginning to ask if the subject had experienced a cold in the past 14 days. To determine whether these modifications of the ACQ altered the conclusions of the manuscript, adult asthmatics $(n=96)$ with a cold were randomly administered the original ACQ in the clinic and then either the original or the ACRN modified ACQ over the telephone. The concordance correlation coefficient between the in-clinic original ACQ and the telephone-administered original ACQ was 0.96 (95\% CI 0.93-0.98). The concordance correlation coefficient between the in-clinic original ACQ and the telephoneadministered modified ACQ was 0.97 (95\% CI 0.95-0.98).

Given the high concordance correlation coefficients in this study, we conclude that the modifications to the ACQ did not alter our conclusion that the severity of a cold measured within the first 2 days was a unique predictor of subsequent worsening of asthma control. Furthermore, the telephone administration of the ACQ does not appear to alter the performance characteristics of this instrument. In the future, we will utilise the original version of the ACQ to assess post-cold asthma control.
M.J. Walter*, M. Castro\#, E. Israel ${ }^{\mp}$ and C.A. Sorkness ${ }^{+}$, on behalf of the National Heart, Lung and Blood Institute's Asthma Clinical Research Network

*Dept of Critical Care Medicine, SSM DePaul Health Center, "Pulmonary and Critical Care Medicine, Washington University School of Medicine, St Louis, MO, USA, "Pulmonary and Critical Care Division, Dept of Medicine, Brigham and Women's Hospital and Harvard Medical School, Boston, MA, and ${ }^{+}$Dept of Pharmacy Practice, University of Wisconsin School of Pharmacy, and Dept of Medicine, University of Wisconsin School of Medicine and Public Health, Madison, WI, USA.

Correspondence: M. Castro, Pulmonary and Critical Care Medicine, Washington University School of Medicine, Campus Box 8052, 660 S. Euclid Ave., St Louis, 63110-1093, MO, USA. E-mail:castrom@wustl.edu

Statement of Interest: None declared.

\section{REFERENCES}

1 Walter MJ, Castro M, Kunselman SJ, et al. Predicting worsening asthma control following the common cold. Eur Respir J 2008; 32: 1548-1554.

2 Walter MJ, Castro M, Kunselman SJ, et al. Author correction: Predicting worsening asthma control following the common cold. Eur Respir J 2009; 34: 1212.

3 Juniper EF, Svensson K, Mork AC, et al. Measurement properties and interpretation of three shortened versions of the asthma control questionnaire. Respir Med 2005; 99: 553-558.

DOI: $10.1183 / 09031936.00054711$

\footnotetext{
${ }^{\#}$ E.F. Juniper is the copyright holder of the Asthma Control Questionnaire. Further information can be found at www.qoltech.co.uk
} 